\title{
An loT and Mobile Cloud based Architecture for Smart Planting
}

\author{
Changbo $\mathrm{Ji}^{1}$, a , Hongyue $\mathrm{Lu}^{2}$, Changqing $\mathrm{Ji}^{2}{ }^{2} \mathrm{~b},{ }^{*}$, Jingguo $\mathrm{Yan}^{2}$ \\ ${ }^{1}$ Agriculture and Biological Technology Institute, Eastern Liaoning University, Dandong 118003, \\ China \\ ${ }^{2}$ School of Physical Science and Technology, Dalian University, Dalian, 116622, China

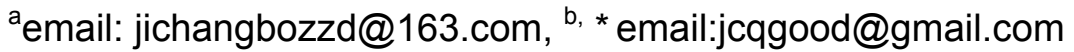

Keywords: Smart Planting; Internet of Things; Cloud Computing

\begin{abstract}
Nowadays, Smart Planting has become a core and an effective technology to achieve agricultural modernization technologies. In this paper, a new scheme of smart planting innovative scheme based on the Internet of Things and Cloud Computing technology is independently developed. We provide an architecture and system model for effective planting management, remote smart control system and application strategies. The perfect combination of them can promote smart planting and effectively solve the problems of farmers' planting.
\end{abstract}

\section{Introduction}

The IoT (Internet of Things) is a hot concept newly emerged in recent years. It is a network of Internet enabled objects, as well as the network services that interact with these objects. Underlying the Internet of Things is technologies such as RFID (Radio Frequency Identification), sensors, and smart devices [1]. Soon after the President of the United States Barack Obama proposed the concept of "smart planet" in 2009, Premier We proposed the development idea of "sensing China", which mainly emphasized the development of IoT and strategic new industries [2].

In the field of Information Technology, the IoT is a technological revolution that represents the future of computing and communications. It refers to a network of objects, and is uaually a self-configuring wireless network. As a whole, in this technical system as shown in Figure 1, the coral technologies mainly include: RFID, sensor, WSN, "3S", mobile smart device and cloud computing etc. The technology of "3S" includes RS (Remote Sense), GIS (Geography Information System) and GPS (Global Position System) [3].

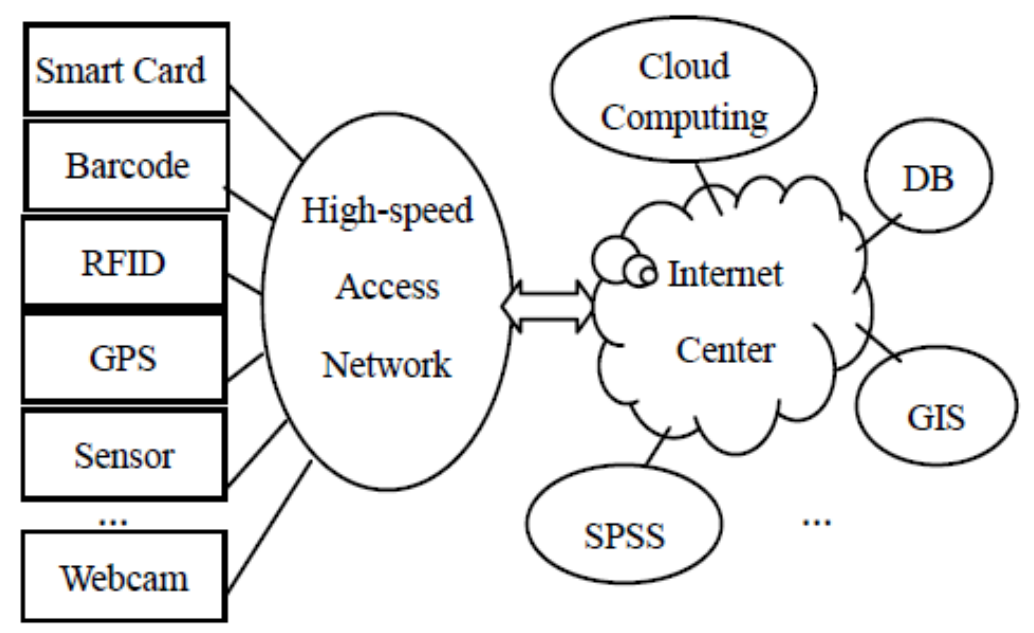

Fig.1. Technology System of IoT

China is a typical big planting country with productivity of fruit, berry, drupes and nut etc. Both at home and abroad, practice shows that information technology (including IoT and Cloud) is the key to develop urban modern planting. Especially IoT brings this fusion into a new stage of intelligent planting, which focuses on perception, interconnection and intelligence [4]. Through the

\footnotetext{
* Changqing Ji is corresponding author.
} 
using IoT related technologies, the efficiency and using-friendly of planting production should be significantly improved. The combination of Radio Frequency Identification, Wireless Sensor Network and planting information has good prospects for development. As an instance, soil moisture sensor, temperature sensor, video surveillance and other information sensing technology are also widely used for monitoring and controlling the growth of plant. In addition, the smart planting logistics information-based network and intelligent development of large scale artificial mining technology application requirements.

In this paper, we propose a smart planting intelligent surveillance system based on the IoT. We propose a planting ontology for the purpose of planting internet of things. The remainder of the paper is organized as follows: Section 2 puts forward the basic conception and the overall design of proposed architecture for effective planting management. In section 3 reference model for smart planting management is discussed. Section 4 concludes the current work and discusses possible future avenues for this research.

\section{Architecture for effective planting management}

(1) Internet of Things

IoT is a kind of intelligent technology, including identification, sensor and intelligence. The definition of IoT as the time changes of cloud computing. It is now defined as IoT $=$ cloud computing + ubiquitous network + intelligent sensing network. Cloud computing management platform is the "brain" of cloud computing. IoT develops to an enormous number of smart tags interacting with and transmitting information to each other and with decentralized and central systems. Ubiquitous network including 3/4G, GSM, WLAN, LTE, RFID, Zigbee, NFC, blue tooth and other wireless communication. It also includes optical cable and other wire communication protocol and technology [5].

(2) Cloud Computing

Cloud computing is associated with new paradigm for the provision of computing infrastructure and big data processing method for various resources. The purpose of cloud computing is to access large amounts of computing power, through the aggregation of resources, and to provide a single system view. Cloud computing is becoming a powerful architecture to perform large-scale and complex computing, and has revolutionized the way that computing infrastructure is abstracted and used. In addition, an important goal of these technologies is to deliver computing as a solution for tackling big data, such as large-scale, multi-media and high dimensional data sets [6].

(3) Challenges for Smart planting

In order to define the feasibility and the wider standards for smart planting, two main challenges must be solved: 1) The lack of integration of current solutions and 2) the unexisting common reference model for smart planting.

We define the following requirements that must be fulfilled to develop a smart planting system:

Request 1: The smart planting system ought to include these planting management and network techniques: identify the planting area of the network resources, remote management of physical equipment and operation of basic units, definition of operations and conditions over the network.

Request 2: It should provide an extensible and flexible architecture integration of various systems. To do that, it must be integrate IoT, Cloud Computing and Mobile systems direct access to individual planting management devices, and also defined open interface among different layers(include communication, process control, application layers, etc.).

Request 3: It should support system model and interoperability with other applications such as expert system, geographic information systems and also databases contain information regarding the soil, weather forecasting, environment, farming, etc. 


\section{System model for smart planting management}

Wireless Sensors Contributing to solutions that integrate the IoT paradigm into planting management processes can be beneficial to address expected solutions. The rest of this Section describes the main benefits of adopting IoT and the IoT characteristics that are considered in the proposed Smart Planting system model.

As we all known, smart agriculture solution refers to precision farming, greenhouse automation and environment monitoring \& control. In many sectors farmers, researchers or greenhouse owners need to measure the degree and extent of certain aspects of environment. As shown in Fig.2, they can also be used on smart planting.

Smart Sensors for forestry and agriculture play an important role today. The need for increasing the production and simultaneously the efforts for minimizing the environmental impact and for saving costs make the sensor systems be the best allied tool. The use of sensors helps to exploit all available resources appropriately and to apply hazardous products moderately. When nutrients in the soil, solar radiation, humidity, density of weeds and all factors affecting the production are known, this gets better and the use of chemical products such as fertilizers, herbicides and other pollution products can be reduced considerably.

Smart Planting IoT interconnects the information sensing devices such as RFID, sensors, GPS system, and two dimensional codes according to pre-determined protocol. It exchanges information and communicates through wired or wireless network. The IoT can achieve functions such as intelligent recognition, data acquisition, intelligent control, location tracing, tracking, monitoring and management [7].

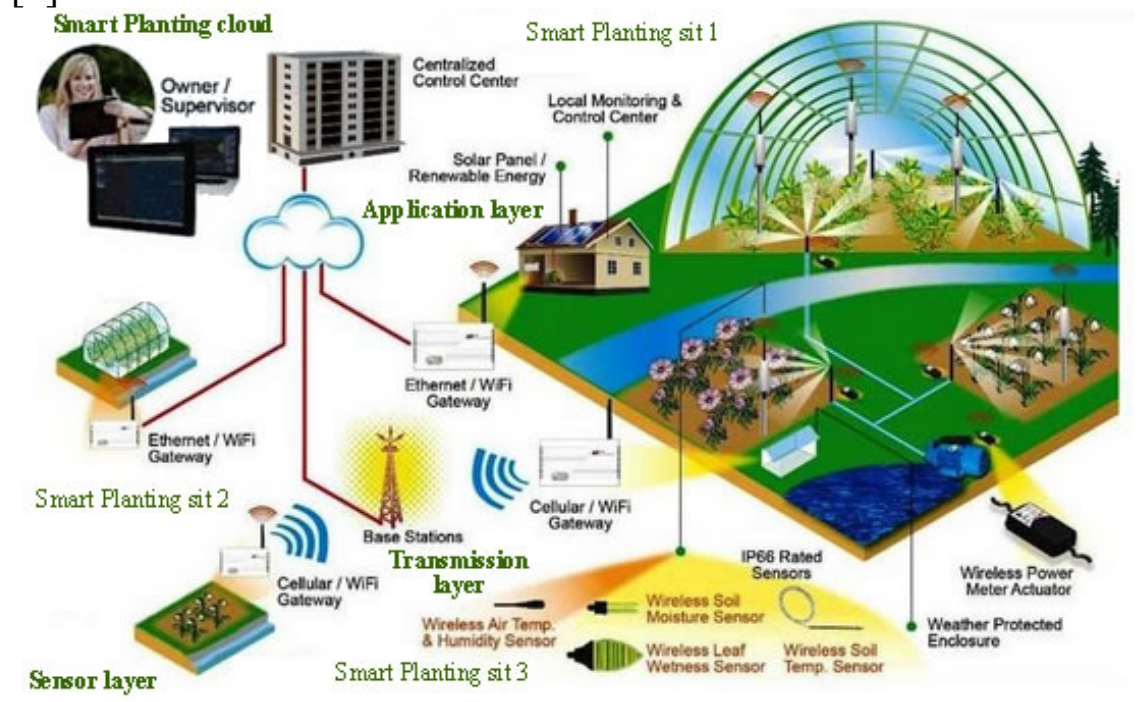

Fig.2. Smart Planting Solution

The proposed architecture of public information service system for smart planting IoT is shown in Figure 2, which is beneficial in any definition of three layers. Smart planting IoT systems are able to execute processes and communicate using a standard communication interface; in the coordination layer, it can be useful to design new coordination applications, with the purpose to orchestrate the management and exploitation layer with the subsystem layers; and finally, in the management and exploitation layer, IoT identification capabilities contribute to provide tailored information services for a specific plant distribution network community.

(1) The sensor layer is responsible for numerical sensor of physical values in agricultural production, relying on the ubiquitous sensor deployment.

(2) The transmission layer summarizes the collected data from sensors, using internet technology and integration of geographic information collection point.

(3) The application layer display and expresses of specific business logic agriculture through interactive interface. 


\section{Remote Mobile Control System in Smart Planting}

We design the remote-control system plan in smart planting. It is possible to communicate between intelligent equipments and remote mobile devices. Varieties of device message can also be utilized for a data exchange with various types of censors. So we can effectively manage and share data through these data source by using our smart planting system.

Remote automatic control system of smart planting is mainly composed of integrated control system, the intelligence terminal devices and the wireless networking system. The integrated control system has some basic elements: heating system, evacuation, air inbleed system, humidification system, sprinkler system and air blower intelligence control system etc. Control software does analysis and statistics, dynamically display the curve of sensor data, carries on the comparison to sensor data and the specified value, when more than a specified value control instruction, automatic spray control, ventilation, heating, humidification androlling equipment work. According to statistical data and dynamical data collection and records on the basis of the analysis of the smart sensors, control software will compare predetermined value and current value dynamically at runtime. If the current value already exceeds the predetermined threshold, a signal will be output to automatic control of spray, ventilation, heating, humidification and rolling equipment. As the current value demands on falling below the threshold level, the resources of that were allocated by control system will be to free up. Customers can also take complete remote control through GRPS/3G network by using smart device (such as pad, phone and pocket pc etc.).

\section{Mobile Cloud Computing Platform}

It is the key step for implementing precision planting to collect timely the spatial-temporal variant information that influences plant production. In order to intelligent monitor the situation of the large scale planting, the precise environment surveillance and the early warning analysis are necessary. Thus, in this section, we analyzed the study and application of Mobile cloud computing and the IoT on smart planting.

First of all, we need continuous data and information gathering, including the environment temperature, concentration of carbon dioxide, air humidity, soil moisture and content of soil NPK etc. We index these data so that the results can be rapidly accessed by mobile user query application. Secondly, we start to compress all the data sets and upload them to the cloud computing center. By using cloud computing database, cloud computing management of relevant record and storing of data related to production performance shown by individual plant and plant groups, analyze and compute, make production plans, etc. Cloud computing is also able to identify the growth of plants by using pattern identification technology and perform dynamic monitor of plant growing with help of other sensing equipment [5]. Finally, front-end mobile service that serves users' online requests for queries. Farm workers can grasp the accurate, real-time state of planting by using mobile device. This mobile system gives researchers a precise view of the growth of plants, effectively reduce the harmful factors such as natural disasters, diseases and insect pests on crop yield, the quality of agricultural products and the influence of soil quality.

\section{Conclusions}

In this paper we presented the system initiative for defining reference architecture for smart planting management based on integrating IoT capabilities to achieve a scalable and feasible industrial system. We define the management exploitation layer, coordination layer, subsystems layer and administration layer and the interfaces that enable layer to interacte. We also consider the system model, which defines the physical elements executing planting management processes in a hierarchical way.

In the future, these systems will actually be further implemented to more planting and agricultural demonstration projects of Liao Ning province in China. The information service will be 
improved to support more types of products and provide more services. By taking advantage of IoT technology, the efficiency of planting production can get a significant improvement. With constantly improving, agriculture IoT must be able to lead agriculture and planting production to a new era.

\section{Acknowledgement}

This work is supported by the Project of College Students' Innovative and Entrepreneurial Training Program (20150056, 20150072, 201411258048 and 201411258010), the general program of Liao Ning Provincial Department of Education Scientific Research (L2014492 and L2014283), the Twelve-Five Teaching-reform Planning Project of Liao Ning Province of China (JG14DB037), Science and Technology Program of Dalian of China (20150280), the PhD Start-up Foundation of Dalian University of China (2015), and the Teaching-reform Project of Dalian University of China (2013122G1 and 2013123G1).

\section{References}

[1] Zhao J, Zhang J, Feng Y, et al. The study and application of the IOT technology in agriculture[C]. Computer Science and Information Technology (ICCSIT), 2010 3rd IEEE International Conference on. IEEE, 2010, 2: 462-465.

[2] Mei Fangquan. "Smart planet and sensing china - analysis on development of IOT" [J]. Agricultural Network Information, Vol.12, pp. 5-7, 2009.

[3] Duan Y E. Research on integrated information platform of agricultural supply chain management based on internet of things[J]. Journal of Software, 2011, 6(5): 944-950.

[4] Sarma A C, Girão J. Identities in the future internet of things[J]. Wireless personal communications, 2009, 49(3): 353-363.

[5] TongKe F. Smart Agriculture Based on Cloud Computing and IoT[J]. Journal of Convergence Information Technology, 2013, 8(2).

[6] Ji C, Li Y, Qiu W, et al. Big data processing: Big challenges and opportunities[J]. Journal of Interconnection Networks, 2012, 13(03n04).

[7] Qiu T, Xiao H, Zhou P. Framework and case studies of intelligence monitoring platform in facility agriculture ecosystem[C]. Agro-Geoinformatics (Agro-Geoinformatics), 2013 Second International Conference on. IEEE, 2013: 522-525. 